\title{
Feedback Control of Decoupled Flatness and Edge drop for the Cold Rolling Mill
}

\author{
Xiaomin Zhou ${ }^{1, a}$, Xiaoxue Yue ${ }^{1, b}$ \\ ${ }^{1}$ School of Mechanical Engineering, University of Science and Technology Beijing, Beijing 100083 \\ aemail: zhouxiaomin@ustb.edu.cn, bemail:542757719@qq.com
}

Keywords: Flatness; Edge Drop; Decoupling Control; Feedback; Cold Rolling Mills

\begin{abstract}
The flatness and edge drop is a strong-coupled multivariable control system, so realizing the decoupling control of them is one of the most important issues to achieve the high-precision control. In this paper, the decoupling feedback controller and servo compensator are proposed to trace the target value of flatness and edge drop. Simulation results show that the flatness and edge drop decoupling feedback control has good dynamic performance to trace the flatness and the edge drop by respective controller.
\end{abstract}

\section{Introduction}

Flatness and edge drop are two significant parts of strip quality. Flatness control precision has satisfied the demand of users, but edge drop control level still has no breakthrough. The edge drop of strip will directly influence the side scrapping of strip and reduce the product yield [1]. In recent years, the edge drop control has become the concern issue in the rolling process control field. The technologies such as K-WRS work roll, EDC work roll etc. are produced one after another [2] [3]. The edge drop is mainly controlled by work roll shift. The work roll shift inevitably influence the shape of loaded roll gap [4] [5]. Due to the inseparable relations between the roll gap and flatness, the flatness must be affected. This shows that the combined control of flatness and edge drop is a coupled multivariable control system. So realizing the decoupling control of flatness and edge drop is one of the most important issues to achieve the strip high precision control. In this paper, the modelling of system and feedback control method of flatness and edge drop decoupling system are analyzed. Simulation results represent that the performance of flatness and edge drop decoupling control is improved by the respective controller of decoupled flatness and edge drop.

\section{Decoupling control of flatness and edge drop}

The coupling relation is strong during controlling the quadratic crown by the work roll bending and controlling the edge drop by work roll shift [6]. Inverse decoupling control realizes the decoupling control of the original system by connecting the inverse system in series before the original system and constituting the pseudo linear composite system [7]. Due to the powerful ability in nonlinear mapped and self-learning of the neural network, neural network inverse decoupling control combining the neural network with inverse system decoupling control will give both methods full play and gain the better decoupling control effect[8] [9]. The static neural network approximates the static nonlinear function and the delay factor or integrators reflect the dynamic characters of the system. The whole system can be changed to the decoupled normalized pseudo linear composite system. It has the linear transmit relation by connecting the neural network inverse system in series before the original system [10].

Flatness and edge drop control model cannot be established precisely, but both of them can be simplified to second order dynamic system since the work roll bending and work roll shift are controlled by the hydraulic cylinder and servo valve [11]. The inputs of the inverse system are the quadratic crown and edge drop which are the outputs of the original system and first, second order derivative of quadratic crown and edge drop. The outputs of the inverse system are work roll bending and work roll shift which are the inputs of the original system. The pseudo linear 
composite system is established by connecting the neural network inverse system and original system in series. The inputs of this system are the first, second order derivatives of the edge drop and the quadratic crown which are connected to static neural network inverse system by four integrators. The outputs of the edge drop and the quadratic crown can be obtained by neural network inverse system and original system. Now, this composite system is decoupled and linearized. Flatness and edge drop has been decoupled to two relative independent system.

For the decoupled system, the flatness and edge drop has been decoupled to two relative independent system, so the feedback controller of flatness and edge drop can be designed independently to trace the respective desired value. The feedback control of the decoupling system is shown as Fig.1.

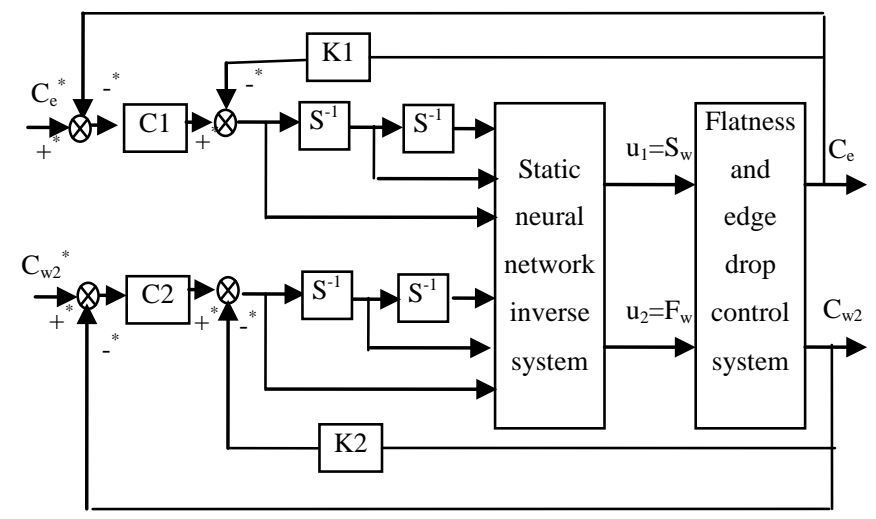

Fig.1 the feedback control principle of the decoupling system

The decoupled flatness and edge drop model are the second order system. So the servo compensator K1 and K2 need to be designed for the stability of system. The form of the K1 and K2 compensator is $\left(\mathrm{k}_{0}+\mathrm{k}_{1} \mathrm{~s}\right)$. C1 and C2 are the controllers to trace the system target.

After the compensation by the $\mathrm{K} 1$ or $\mathrm{K} 2$ compensator, the transfer function of pseudo linear open loop system is as equation (1) shown as follows.

$$
\mathrm{G}(\mathrm{s})=\frac{1}{\mathrm{~s}^{2}+\mathrm{k}_{1} \mathrm{~s}+\mathrm{k}_{0}}
$$

To make sure the error of system zero, the controller C1 or C2 need to be chosen as equation (2) shown as follows.

$$
\mathrm{C}=\frac{\mathrm{a}_{0}+\mathrm{a}_{1} \mathrm{~s}}{\mathrm{~s}}
$$

The transfer function of pseudo linear close loop system is as equation (3) shown as follows.

$$
\emptyset(s)=\frac{P C}{1+P C}=\frac{a_{0}+a_{1} s}{s\left(s^{2}+k_{1} s+k_{0}\right)+a_{0}+a_{1} s}
$$

To choose reasonable parameters such as $\mathrm{k}_{0}, \mathrm{k}_{1}, \mathrm{a}_{0}, \mathrm{a}_{1}$, the transfer function of close loop system can be obtained as equation (4) shown as follows.

$$
\emptyset(s)=\frac{\omega_{1}^{2}\left(s+\delta_{1}\right)}{\left(s+\delta_{1}\right)\left(s^{2}+2 \xi_{1} \omega_{1} s+\omega_{1}^{2}\right)}
$$

The term of $s+\delta_{1}$ is to realize the zero-pole cancellation. The term of $\xi_{1}$ is the damping ratio. And the term of $\omega_{1}$ reflects the system response speed.

\section{The simulation and analysis}

The edge drop trace control curve of the decoupling feedback control system is as Fig.2 shown as follows. The edge drop target value is stepped from 20um to 0um when time is 0.5 second. The edge drop and flatness output is shown as Fig.2 (a) and Fig.2 (b). It can be seen that the actual edge drop traces from 20um to oum controlled by the decoupling feedback control system, but the flatness nearly has no influence. 


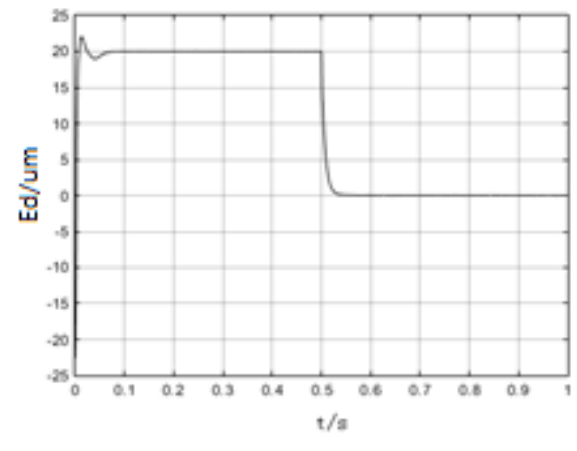

(a) edge drop output

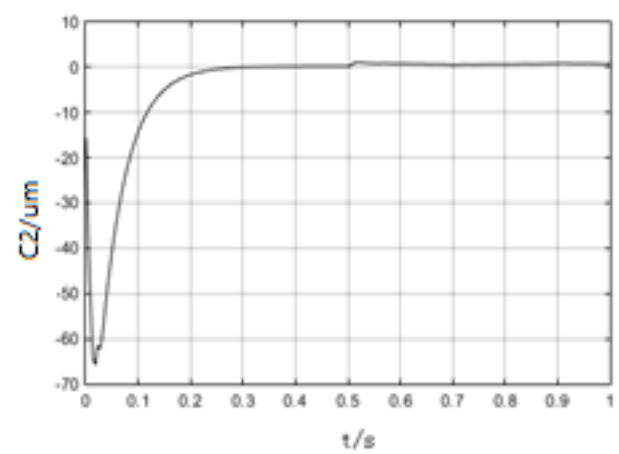

(b) flatness output

Fig.2 The edge drop trace control of the decoupling feedback control system

The flatness trace control curve of the decoupling feedback control system is as Fig.3 shown as follows. The flatness target value is stepped from 30um to 0um when time is 0.5 second. The edge drop and flatness output is shown as Fig.3 (a) and Fig.3 (b). It can be seen that the actual flatness traces from 30um to 0um controlled by the decoupling feedback control system, but the edge drop nearly has no influence. Simulation results show that the decoupling feedback control system has a good decoupling and trace effect of the edge drop and the flatness. And the system has good dynamic performances such as the quick response and small overshoot.

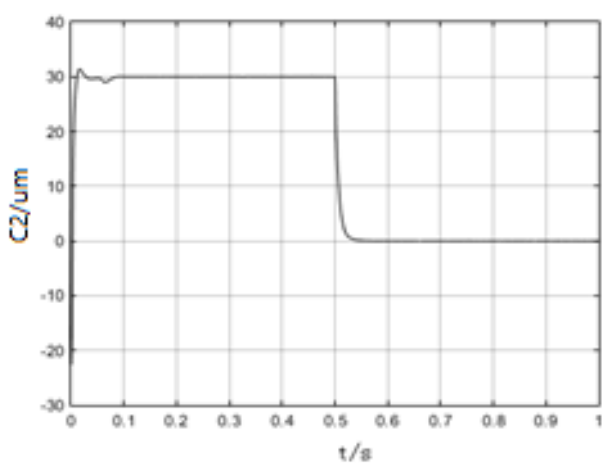

(a) flatness output

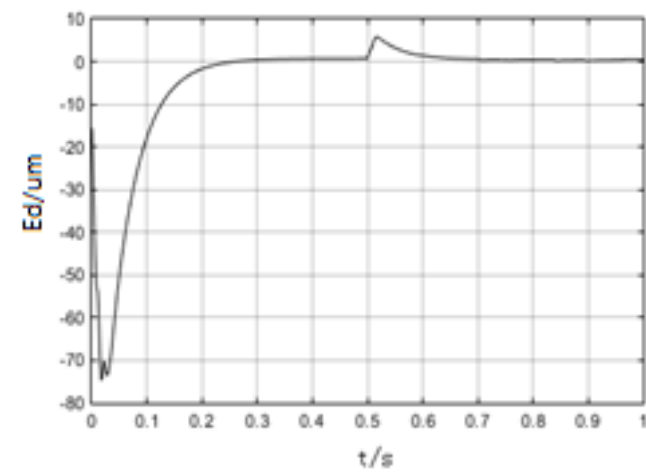

(b) edge drop output

Fig.3 The flatness trace control of the decoupling feedback control system

\section{Conclusion}

The strong coupling relation exists between the flatness and edge drop control. The decoupling control is completed by combining the inverse system with the original system into pseudo linear composite system. The servo compensator and the feedback controller are designed for the decoupling control system. Simulation results show that the decoupling control system has a good decoupling and trace effect of the edge drop and the flatness. And the system has good dynamic performances such as the quick response and small overshoot.

\section{Acknowledgement}

In this paper, the research was sponsored by the National Natural Science Foundation of China (Project No. 51204017).

\section{References}

[1] Yang G H, Zhang J, Cao J G,et al. Edge Control Technology of 4-hi and 6-hi Tandem Cold Rolling Mills[J]. Metallurgical Equipment, 2009.(178):1-5.

[2] Lu H T, Cao J G, Zhang J, et al. Edge drop control of a taper roll during continuous cold 
rolling[J]. Journal of University of Science and Technology Beijing, 2006, 28(8):774-777.

[3] Zhou X M, Zhang Q D, Wu P. The Summary of the Strip Edge Drop Control Technology [J]. ShangHai Metals. 2007,29(1): 21-24.

[4] Yang G H, Cao J G, Zhang J, et al. Comprehensive Control for Strip Edge Drop, Crown and Flatness on Tandem Cold Rolling Mill[J]. Metallurgical Equipment. 2008.(172):1-5.

[5] Xiaomin Zhou, Xiaoxu Cheng. The Analysis of Influence on the Edge Drop of Tandem Cold Rolling Mill. Applied Mechanics and Materials. 2014.Vols. 548-549

[6] Zhou X M, Zhang Q D, Wang C S, et al. Edge Drop and Flatness Decoupling Control on UCMW Cold Mill[J]. Iron and Steel. 2007,42(5):55-57.

[7] Hong Y Z, Zhu H Q, Wu Q H, et al. Dynamic Decoupling Control of AC-DC Hybrid Magnetic Bearing Based on Neural Network Inverse Method [A]. International Conference on Electrical Machines and Systems [C]. Zhenjiang, 2008:3940-3944.

[8] Wang Dongcheng. A Model Coupling Method for Shape Prediction [J]. Journal of Iron and Steel Research, International, 2012,19(6):19-24.

[9]Abdelkhalek S, Montmitonnet P, Legrand N, et al. Coupled Approach for Flatness Prediction in Cold Rolling of Thin Strip[J]. Int J Mech Sci, 2011,53: 661-675.

[10] Song Y X, Ma J L, Zhang H X, et al. The Decoupling Control of Induction Motor Based on Artificial Neural Network Inverse System Method. International Conference on Electrical and Control Engineering [C]. 2010:2287-2291.

[11] Peng P. Research on Coupling Relations and Decoupling Control for Wide Strip Tandem Cold Mill. [D].Beijing: University of Science and Technology Beijing, 2008. 